\title{
Crise morale et crise éducative en Afrique
} subsaharienne

Jean-Paul Ngoupandé

\section{CpenEdition}

1 Journals

Édition électronique

URL : http://journals.openedition.org/ries/4170

DOI : $10.4000 /$ ries. 4170

ISSN : 2261-4265

Éditeur

Centre international d'études pédagogiques

\section{Édition imprimée}

Date de publication : 5 mars 1995

Pagination : 119-132

ISSN : 1254-4590

\section{Référence électronique}

Jean-Paul Ngoupandé, "Crise morale et crise éducative en Afrique subsaharienne », Revue internationale d'éducation de Sèvres [En ligne], 05 | 1995, mis en ligne le 16 mars 2015, consulté le 14 novembre 2019. URL : http://journals.openedition.org/ries/4170 ; DOI : 10.4000/ries.4170

Ce document a été généré automatiquement le 14 novembre 2019.

(c) Tous droits réservés 


\title{
Crise morale et crise éducative en Afrique subsaharienne
}

\author{
Jean-Paul Ngoupandé
}

\section{Une dérive morale inquiétante}

1 Dans de nombreux pays d'Afrique francophone, les universités ont été le lieu, ces dernières années, d'actes de violence : agressions, enlèvements, lynchages, « braisages ${ }^{1}$ ». Beaucoup d'entre elles sont devenues le champ clos d'affrontements, de guérilla quasi permanente.

2 Pour illustrer le désarroi dans lequel a plongé la jeunesse africaine scolarisée, il faut évoquer les grèves à répétition conduisant, souvent, à des années entières sans scolarité, que l'on appelle désormais, partout, des années «blanches». Pour le seul mois d'avril 1994, au moins dix des quatorze anciennes colonies françaises d'Afrique subsaharienne ont connu des arrêts de cours dans leurs universités. En réalité, au cours de la dernière décennie, aucun de ces pays n'a passé une année scolaire et universitaire normale: grèves, affrontements dans la rue, occupations de locaux, saccages de bureaux, séquestrations, molestation de responsables, destructions de véhicules ont rythmé le déroulement d'années scolaires dont le bilan ressemblait le plus souvent à celui de guerres civiles à peine larvées.

3 Le bilan pédagogique, quant à lui, est facile à établir : rares sont les universités qui totalisent plus de dix semaines effectives d'enseignement dans l'année.

4 Cela se traduit dans un indicateur encore plus parlant : la baisse constatée de niveau, partout déplorée, mais nulle part en situation d'être stoppée. Les motifs du déclenchement de ces troubles vont des résultats des examens aux questions liées aux bourses, en passant par les situations les plus insolites, tel cet étudiant blessé dans une banale bousculade entre passagers en train de monter dans un bus aux heures de pointe, ou encore cet autre, motocycliste, renversé par une voiture...

5 C'est vrai que les bourses constituent, notamment depuis la dévaluation du franc CFA, la raison principale des manifestations, grèves et troubles. Mais le foisonnement des 
causes, y compris les plus inattendues, est tel qu'il convient de les prendre en réalité pour ce qu'elles sont aujourd'hui : l'arbre qui cache la forêt.

6 C'est de cette forêt qu'il importe de débattre sérieusement, parce que les réponses éparses à des revendications apparemment aussi hétéroclites ne sauraient tenir lieu de thérapeutique réelle face à un mal qui doit être considéré comme profond.

\section{Les racines du mal}

7 Ce mal plonge ses racines, on le sait, dans l'histoire des systèmes de formation hérités de la période coloniale. Ayant une vocation essentiellement administrative, c'est-à-dire destinée à fournir rapidement les cadres administratifs qui faisaient cruellement défaut aux nouveaux États indépendants, l'école africaine postcoloniale a accompli cette mission à peu près correctement, à la satisfaction de tous les partenaires du système : l'État recruteur, les familles, les formateurs et les produits eux-mêmes. C'était la belle époque où les diplômés étaient parfois intégrés à la fonction publique avant même d'avoir passé leurs derniers examens, où l'État exigeait des «engagements décennaux ", pour être sûr que les bénéficiaires de bourses nationales n'iraient pas ailleurs que dans l'administration publique.

8 L'âge d'or de l'école à vocation administrative dura environ deux décennies. Mais déjà, vers le milieu des années soixante-dix, l'essoufflement du dispositif d'absorption devenait perceptible.

Quelques acteurs avisés du système éducatif tirèrent la sonnette d'alarme dans certains pays. Des solutions furent même proposées pour tenter de briser la logique infernale de la machine à fabriquer des fonctionnaires. En Centrafrique, l'expérience la plus audacieuse fut celle des écoles de promotion collective (EPC). Dans l'esprit de ses promoteurs, cette nouvelle école était appelée à s'intégrer parfaitement au monde rural, et donc à préparer les jeunes de la brousse aux petits métiers susceptibles de redonner vie aux villages, et de concourir au passage progressif de l'agriculture traditionnelle, d'autosubsistance familiale, à une économie paysanne moderne tournée vers le marché. Ces petits métiers allaient de la fabrication et de l'entretien du petit outillage agricole à la menuiserie en passant par la vannerie et d'autres activités directement liées aux productions spécifiques des régions concernées. Au terme du cycle primaire, les sortants des EPC étaient destinés à animer la vie économique des villages. Le contenu de l'enseignement prenait donc largement en compte les impératifs de cette réinsertion dans le milieu traditionnel, en premier lieu l'utilisation de la langue sango ${ }^{2}$.

10 Malgré ses imperfections, cette expérience offrait une issue autre que l'engorgement des filières d'enseignement général conduisant au fonctionnariat après les études secondaires et supérieures. Elle fit pourtant long feu. Les mentalités ne s'y prêtaient guère. Le mythe de la réussite en col blanc, solidement ancré chez les parents d'élèves, provoqua une désaffectation rapide. Pour les paysans, « le retour à la terre » préconisé par les EPC était vécu comme un échec. La promotion sociale, la vraie, se traduisait, depuis toujours pour eux, depuis l'époque coloniale, par les trois indicateurs qu'étaient : la bonne maîtrise de la langue française, le départ du village vers la ville et l'emploi dans un «bureau », la rémunération important peu. 
11 C'est donc tout naturellement qu'ils prirent eux-mêmes l'initiative de retirer leurs enfants de "ces écoles où l'on enseigne en sango ». L'idée que la véritable promotion signifie la rupture avec le travail manuel était si forte que l'administration scolaire ellemême la véhicula à sa façon, avant et après l'indépendance, en ce qui concerne l'enseignement technique et professionnel. L'orientation vers ce type d'enseignement était généralement considérée comme la sanction d'un échec: ce sont ceux qui ne réussissaient pas dans le secondaire général qui étaient « dirigés ", plutôt de force que de gré, vers le collège technique. Il était de bon usage de repérer, dès la sixième, les meilleurs éléments, à orienter d'office vers la filière-reine, dite "classique», qui comportait l'étude du latin, puis du grec à partir de la quatrième ${ }^{3}$.

\section{Une irresponsabilité criante}

12 Les indicateurs avaient, dès 1973, révélé les déficiences fondamentales des appareils économiques africains postcoloniaux. En effet, le renchérissement des prix de l'or noir consécutif à la guerre du Kippour (octobre 1973) avait mis à nu l'extrême fragilité de structures de production basées sur la monoculture de quelques produits de rente (coton, café, cacao) et la part excessive prise par le secteur étatique, fournisseur quasiexclusif des emplois modernes.

13 Du jour au lendemain, des économies entièrement assistées, peu habituées à affronter les fluctuations du marché et les adaptations rapides qu'elles nécessitaient, manifestèrent des signes plus qu'inquiétants d'inadaptation et d'inefficacité, sur lesquels au demeurant l'agronome français René Dumont avait, une décennie plus tôt, vivement attiré l'attention dans ce qu'il faut bien appeler aujourd'hui son livre prophétique, L'Afrique noire est mal partie.

Deux «mirages » masquèrent pourtant, une fois de plus, la gravité de la situation et l'urgence d'entreprendre des réformes véritables, nécessairement douloureuses, pour stopper la dérive économique et ses conséquences sociales, notamment le chômage massif des jeunes. Ce fut d'abord l'aubaine, pour quelques pays africains producteurs d'or noir, de l'afflux massif de pétrodollars.

15 Les pétrodollars croulant dans les banques occidentales cherchaient des projets à financer, nonobstant le sérieux et la fiabilité de ceux-ci. Ce fut la grande époque des "éléphants blancs » et des commissions «longues comme le bras ", qui aggravèrent l'endettement du continent. Le réveil fut brutal lorsque survint le second choc pétrolier, à la fin des années soixante-dix, et que s'installa la crise économique aux allures de catastrophe irrémédiable qui dure depuis une quinzaine d'années.

Les générations futures d'Africains s'interrogeront sans doute longtemps sur le véritable sens à donner aux rendez-vous manqués du développement dans les années soixante et soixante-dix. Ce débat, qui commence à poindre, et où l'on perçoit enfin un début de regard non complaisant des Africains sur eux-mêmes ${ }^{4}$, devra situer clairement les responsabilités des élites africaines, et noter les tares propres aux sociétés africaines, au lieu du traditionnel "c'est la faute aux colonisateurs", qui nous a jusqu'ici servi d'alibi confortable.

17 En attendant, et pour revenir à notre sujet, il importe de s'arrêter sur l'irruption de l'ajustement structurel dans le quotidien des Africains et ses conséquences sur l'évolution des systèmes éducatifs de l'Afrique subsaharienne. 


\section{Le choc des années « $\mathrm{PAS}^{5}$ »}

18 Le début des années quatre-vingt fut marqué, dans plusieurs pays, par une innovation qui éclata comme un tonnerre dans un ciel de saison sèche. Le recrutement automatique dans la Fonction publique n'était plus assuré aux élèves à l'issue de leurs études dans certaines grandes écoles. Pour la première fois était posé le principe de concours, c'est-à-dire d'une compétition, en plus du diplôme de fin d'études, pour accéder à des places dont le nombre, désormais limité, devrait être fixé chaque année, en fonction des besoins. Et comme pour signifier encore plus clairement la fin d'une époque, des mesures réglementant le régime des bourses furent annoncées. La plus spectaculaire de ces mesures fut l'autorisation d'un seul redoublement au premier cycle pour les boursiers ; décision qu'on peut considérer comme peu sévère aujourd'hui mais qui, dans le contexte de l'époque, était une véritable révolution, dans la mesure où des étudiants boursiers pouvaient passer jusqu'à six ans pour obtenir un DEUG, en bénéficiant de dérogations successives pour conserver leur bourse ${ }^{6} .$.

19 Au départ donc se trouve la demande impérieuse, adressée par les institutions de Bretton Woods aux gouvernements africains, de procéder à des réformes macroéconomiques. Celles-ci conditionneraient désormais l'aide financière du Fonds monétaire international et de la Banque mondiale. Les États africains subsahariens avaient d'autant moins le choix que les échéances de la dette accumulée pendant les périodes folles, ajoutées à la raréfaction des ressources financières dues à une brutale chute, à partir de 1980, des cours des principales matières premières, ainsi qu'à la gabegie et à l'imprévision ambiantes, limitaient singulièrement la marge de manœuvre des ministres des finances.

20 Tout le système mondial de l'aide au développement, aussi bien bilatérale que multilatérale, se rangeait désormais sous la bannière des institutions précitées. Leur veto avait valeur de «non » universel pour tous les autres «bailleurs de fonds », tandis que leur accord sur un programme ouvrait la porte aux financements. Ces programmes, baptisés « programmes d'ajustement structurel » (PAS) présentaient partout les mêmes injonctions :

- réduction drastique des effectifs de la fonction publique et limitation des recrutements nouveaux à une arrivée pour trois départs dans le but de diminuer la masse salariale qui représentait souvent 75 à $95 \%$ des budgets nationaux ;

- freinage des dépenses sociales, ce qui signifiait d'abord la diminution sévère des charges liées aux bourses, aux internats, aux restaurants universitaires, pour ne citer que le cas du secteur éducatif;

- privatisation des entreprises publiques et parapubliques devenues des gouffres financiers, le passage au privé se traduisant par des licenciements massifs et le blocage de tout nouveau recrutement: les plus irrécupérables de ces entreprises publiques devront être tout simplement liquidées ;

- priorité absolue au remboursement de la dette, dont le service annuel pouvait représenter jusqu'à $50 \%$ du budget, contraignant les États à négocier des rééchelonnements (Club de Paris pour les créanciers publics et Club de Londres pour la dette d'origine privée ${ }^{7}$ ).

21 Les PAS furent vécus comme un traumatisme par une Afrique subsaharienne dont les élites urbaines avaient été profondément marquées par deux décennies de facilité, d'insouciance, de laxisme et de gabegie. Et comme un malheur ne vient jamais seul, 
1983 fut également l'année de la grande sécheresse, avec son cortège de récoltes compromises et de famines à grande échelle. Elle fut aussi, il est bon de le rappeler, le début de la diffusion du SIDA, dont l'expansion fulgurante pose aujourd'hui le problème même de la survie de ce continent. De cette terrible année 1983 date sans doute ce sentiment d'impuissance devant le sort et ce retour massif à l'irrationnel et au surnaturel dont témoigne la profusion sans précédent de sectes religieuses, de mages et de " prophètes » auxquels la majorité des Africains font aujourd'hui plus confiance qu'à leurs gouvernements.

\section{Le prix du progrès}

Arrêtons-nous un instant sur la signification historique de ces programmes d'ajustement structurel. Une approche facile et réductrice voudrait que leur application, à partir du début des années quatre-vingt, soit la cause principale, et même exclusive, des malheurs présents du continent noir. Les PAS ne sont ni innocents, ni indemnes de reproches, et pour notre part, nous avons eu l'occasion d'en indiquer quelques limites ${ }^{8}$.

Mais il ne faudrait pas que le thermomètre soit accusé à la place de la maladie. Les mesures d'ajustement structurel ont été surtout un révélateur de la profondeur de la crise africaine, crise à multiples facettes, dans laquelle la position du facteur humain nous paraît toucher à l'essentiel. Les PAS, sans vraiment remédier au mal, quoi qu'en disent les experts du FMI et de la Banque mondiale, auront au moins eu le grand mérite de mettre à nu le malentendu originel entre l'Afrique noire et le monde moderne. En contraignant les dirigeants africains à une sorte de glasnost économique avant la lettre, les réformes macro-économiques demandées par les institutions de Washington ont permis de jeter une lumière crue sur les désarticulations fondamentales des sociétés africaines postcoloniales, désarticulations qui expliquent leur incapacité à s'arrimer au wagon de la modernité. Le malentendu originel dont nous venons de parler, et qui dure encore aujourd'hui, repose sur le fait que nous, Africains, et particulièrement les élites, n'avons pas compris que l'accès à la modernité se paye en efforts douloureux. « Il n'y a pas - comme nous avons eu l'occasion de le dire - d'accès doux à la modernité ${ }^{~ ", ~ e n ~}$ commentant la récente dévaluation du franc CFA.

Tous les pays qui ont enregistré des progrès significatifs au cours de ce siècle, tous ceux qui sont passés de la condition de nations sous-développées et assistées à celle d'États économiquement performants et donc maîtres, pour l'essentiel, de leur destin, ont dû payer le prix fort, en privations, en sacrifices, en efforts soutenus, bref, en mesures drastiques de correction de leur trajectoire. La valorisation extrême du travail et de l'effort, de la responsabilité individuelle et collective quant à la préparation de l'avenir commun, a été un leitmotiv pour toute une génération de Japonais, de Sud-Coréens, de Taïwanais, de Malais, etc., qui ont refusé de considérer le sous-développement économique comme une fatalité.

Comparativement à ces nations qui ont choisi de prendre leur destin en mains, celles d'Afrique, depuis l'indépendance, ont surtout opté pour la facilité. Une vision essentiellement ludique de la modernité nous a conduits à privilégier excessivement les solutions ne nécessitant pas d'efforts importants. Ainsi en fut-il de l'éducation et de la formation. L'administration publique suffisait pour résorber la masse en diplômés que nous formions avec de moins en moins de rigueur. 


\section{Le laxisme chronique des systèmes éducatifs africains}

système: tous ceux qui ont pratiqué les systèmes éducatifs des pays d'Afrique subsaharienne francophone savent que, bien souvent, le mot « examen » ne veut plus rien dire. Du primaire aux facultés, les résultats des contrôles de connaissance sont rarement exempts d'irrégularités, faisant même l'objet de négociations où élèves et enseignants et même parents finissent par banaliser la fraude ${ }^{10}$, la malhonnêteté et l'inutilité de l'effort.

La banalisation de la tricherie et du laxisme peut être datée, et il est singulier de constater que, bien souvent, elle fut contemporaine - n'ayons pas peur de le souligner de l'achèvement du processus d'africanisation des cadres du système. Au risque de choquer, nous rappelons que l'école de l'époque coloniale ne transigeait pas avec la tricherie. À cette école-là, on peut reprocher l'inadaptation, l'orientation coupée des nécessités du développement économique. Mais l'objectivité oblige à reconnaître qu'elle cultivait le sens du travail bien fait et l'obligation d'effort. Cet esprit de sérieux et de rigueur se maintint à peu près jusqu'au milieu des années soixante-dix. La glissade accélérée vers le laxisme, à partir de là, interpelle directement les cadres nationaux, responsables administratifs, enseignants et parents ${ }^{11}$. Elle indique que là comme ailleurs, nous avons nié la nécessité de l'effort, et encouragé les marchandages.

\section{La vraie nature de la crise scolaire africaine}

Ces remarques nous ramènent à la question de la nature de la crise scolaire africaine que décrivait très judicieusement, il y a trois ans, au cours d'une conférence de presse à Abidjan, l'ancien ministre ivoirien de l'enseignement supérieur, Alassane Salif Ndiaye : «À y regarder de près, il n'y a peut-être pas, disait-il, une crise de l'école, mais une crise de la société, que l'école reflète plus crûment que d'autres secteurs ».

Qu'est-ce à dire? Tout simplement qu'il faut se garder d'enfermer le débat sur l'école africaine postcoloniale dans un carcan qui ne prendrait pas en compte son environnement historique, au risque de passer à côté de l'essentiel. La crise de l'école africaine postcoloniale est inséparable du refus, conscient ou non, des sociétés africaines contemporaines et notamment de leurs élites, d'accepter comme telles les conditions de passage à la modernité exigées par le monde contemporain. Le siècle qui s'achève a été marqué par des mutations décisives dans la configuration de l'économie mondiale. La plus importante de ces mutations est la mondialisation de l'économie de marché et l'émergence d'une situation de concurrence dont l'âpreté n'a pas de précédent dans l'histoire de l'humanité. Le facteur humain est devenu décisif. Ce qui fait désormais la différence, c'est la qualité des hommes, leur créativité, leur capacité d'adaptation aux situations les plus inattendues, leurs dispositions à saisir et à rentabiliser toutes les opportunités, en un mot, leur aptitude à se surpasser, à faire mieux que leurs rivaux. De telles qualités humaines n'émergent que dans les sociétés qui cultivent l'effort, le goût du travail bien fait et du dépassement de soi ; des sociétés qui valorisent l'excellence au lieu de se complaire dans la médiocrité; des sociétés, 
enfin, qui développent le sens de la responsabilité au lieu d'entretenir la mentalité d'éternels assistés.

Ce qui est en jeu aujourd'hui, en Afrique, au-delà du désarroi d'une jeunesse qui se sent trahie par les aînés, qui n'a plus foi en l'avenir, c'est l'aboutissement logique des choix de facilité qui furent les nôtres dans les années qui ont suivi l'indépendance. Ce qui est en cause, c'est la désastreuse conséquence du renoncement à l'effort, à la rigueur et à la responsabilité. Toutes nos activités se ressentent aujourd'hui de cette option: l'agriculture africaine, incapable d'assurer une véritable autosuffisance alimentaire, parce que très tôt sacrifiée sur l'autel de l'exode rural et de l'importation massive solution de facilité - de produits vivriers ; l'industrie, qui n'a pu prendre un véritable essor, gênée dès le départ par l'extraversion précoce de la consommation, le goût exagéré du luxe cultivé par les élites urbaines, leur préférence affichée pour les produits importés et, par-dessus tout, le parasitisme et la corruption inhérents à ces unités industrielles majoritairement étatisées, devenues de ce fait des rentes pour leurs heureux dirigeants; nos administrations publiques, rendues inefficaces et prévaricatrices parce qu'ayant érigé le passe-droit et le laxisme en normes de fonctionnement, à l'exclusion de toute obligation de résultat.

\section{L'acuité du problème éthique}

Dans un tel environnement, l'école ne pouvait pas connaître une évolution différente. Elle paye aujourd'hui, au prix fort, les inconséquences et le laisser-aller qui l'ont conduite à distribuer à profusion des diplômes de formation supérieure sans rapport ni avec le niveau réel des connaissances, ni, surtout, avec les besoins de l'économie des pays concernés. La voie royale de promotion sociale qu'elle fut est devenue aujourd'hui un chemin de dupes, et la rage avec laquelle les jeunes cassent et détruisent au moindre prétexte traduit ce sentiment de trahison que nous évoquions plus haut; trahison, à leurs yeux, des aînés qui ont assuré leurs arrières puis ont fermé, sous l'injonction du FMI et de la Banque mondiale, la porte d'entrée de la Fonction publique sans proposer une autre issue. Ce n'est pas par hasard si les cibles privilégiées des coups de mains des étudiants en rébellion contre la société sont, le plus souvent, les voitures de l'administration : elles symbolisent le monde dont ils se sentent exclus. Les chances de trouver un emploi dans le système formel, à la sortie des études universitaires, qui étaient de $100 \%$ il y a à peine quinze ans, sont aujourd'hui inférieures à $50 \%$ pour la plupart des filières, et quelquefois bien moins.

Désarroi, perte de foi, crise de confiance, nihilisme : il n'est pas exagéré de parler de crise morale profonde, dès lors que cette jeunesse désabusée ne trouve nulle part, dans son environnement social, des repères moraux susceptibles de l'orienter. Ces actes de violence gratuite, évoqués en introduction, ne sont compréhensibles que si on les replace dans leur contexte éthique. Globalement, nous, Africains, avons sans doute surestimé le ciment moral de nos sociétés, ainsi que leur capacité à endiguer les débordements consécutifs à notre contact avec la modernité. Le tourbillon de la consommation de biens modernes, encouragée par l'urbanisation rapide et la montée en puissance d'élites essentiellement administratives, c'est-à-dire improductives et même parasitaires, a bousculé, dans le plus grand désordre, nos us et coutumes qui étaient historiquement de type rural. 

en organisant ici et là des " concertations nationales ", des " séminaires nationaux » et des "états généraux». Tous nos pays ont sacrifié à ce rite, et souvent à plusieurs reprises depuis une quinzaine d'années. Des diagnostics souvent justes ont été formulés et des réformes parfois courageuses ont été proposées. Mais la plupart des mesures retenues en sont restées au stade des intentions. catégoriels à court terme neutralisent toute possibilité d'action énergique. Chaque catégorie sociale concernée campe sur ses positions avec un égoïsme déconcertant élèves et étudiants arc-boutés sur la revendication de la bourse à tout le monde et sans condition; enseignants moins mobilisés par la dérive du système que par le paiement des heures complémentaires, par exemple; parents n'ayant d'autre souci que le maintien à tout prix de leurs enfants dans le système, même si visiblement ils n'en ont pas les aptitudes; gouvernants soucieux avant tout de la "paix » sur les campus, et prêts à tout concéder y compris en piétinant les exigences de rigueur pédagogique, du moment que les étudiants, redoutés par-dessus tout, se calment.

Cette dernière situation a pris une tournure tragique avec l'expérience du multipartisme en cours depuis cinq ans. Les campus sont devenus des enjeux politiques au point que l'université, et de façon générale, le système éducatif, apparaissent comme les otages des partis politiques. Chaque faction politique y organise «ses " étudiants. Tout se passe, dans certains pays, comme si l'essentiel du débat politique se déroulait à l'université. Partout, les oppositions tentent de mobiliser les étudiants et les élèves, faute de pouvoir le faire dans d'autres catégories de la population, pour faire pression sur les pouvoirs en place, tandis que ceux-ci, pour desserrer l'étau, cèdent sur les mesures rendues pourtant nécessaires par la dégradation de la situation. Les réformes indispensables, qui passent nécessairement par des décisions vigoureuses et impopulaires, sont renvoyées aux calendes grecques. De ce fait, les surenchères 
partisanes occultent le vrai débat sur le naufrage du système et les risques graves que cela comporte pour l'avenir.

Les partenaires extérieurs de l'Afrique, bilatéraux et multilatéraux, ont commencé à se désengager du secteur de l'enseignement supérieur, las d'attendre les réformes qui ne viennent pas. Des voix s'élèvent pour réclamer l'autorisation d'ouverture d'établissements d'enseignement supérieur de statut privé. Le sujet, totalement tabou il $\mathrm{y}$ a à peine cinq ans, commence à être débattu publiquement.

Cette voie pourrait permettre d'offrir une autre perspective et l'on peut espérer que, la concurrence jouant, les universités d'État pourraient être forcées de se remettre en cause. Les réserves des adversaires de la privatisation portent sur la sélection par l'argent qu'instaurerait un système d'universités payantes réservées aux enfants des nantis. Mais cette sélection par l'argent est déjà là puisque, face à la dégradation de l'enseignement supérieur national, il y a belle lurette que des parents, y compris ceux ayant des revenus modérés, ont choisi de se saigner pour payer des études au prix fort en Europe, aux États-Unis et au Canada notamment.

40 À tous les degrés de l'enseignement, les établissements publics sont déjà devenus des dépotoirs, que désertent tous ceux qui ont les moyens de le faire.

\section{L'Afrique au pied du mur}

41 Privé ou pas privé, en tout état de cause, le véritable débat est celui de la capacité des Africains, de leurs élites et de leurs États, à préparer l'avenir commun. Nous avons souligné plus haut l'âpreté de la compétition économique qui caractérise le monde contemporain. Le siècle prochain renforcera, à n'en point douter, cette tendance. Il n'y aura pas de pitié pour les canards boiteux. La qualité des hommes, leur capacité à affronter la concurrence seront, de plus en plus, le facteur décisif du progrès.

Si la régression qualitative actuelle des systèmes d'éducation et de formation des pays d'Afrique noire se poursuit, la marginalisation économique et technologique, déjà avérée, deviendra irrémédiable. Le continent africain, dont la part dans le commerce mondial ne représente que $2 \%$ à peine ${ }^{12}$ et qui n'a déjà plus voix au chapitre, se condamnera à l'insignifiance, s'il ne réajuste pas rapidement, vigoureusement et courageusement sa politique de formation.

Déjà, à la pitié que ses malheurs suscitaient jusqu'ici, succède désormais une indifférence dont les signes sont apparus au début des tragiques événements du Rwanda, en avril dernier.

Des systèmes de formation qui cultivent la malhonnêteté, la tricherie, l'habitude du moindre effort et le laxisme, ne peuvent que conduire tout droit à la catastrophe, c'està-dire au renforcement du discrédit de notre continent, de son isolement et de sa régression.

Nous avons eu l'occasion de dire, en octobre 1993 à Angers, à la réunion de la Task Force des bailleurs de fonds pour l'éducation en Afrique, qu'il est temps que les Africains acceptent enfin d'aborder frontalement leurs problèmes, au lieu de biaiser continuellement, de rechercher des responsables hors du continent. Il y a aujourd'hui des mesures d'assainissement qui ne nécessitent pas de ressources financières nouvelles. C'est d'abord sur notre capacité à les prendre et à les appliquer fermement que nous serons jugés. Car si nous ne sommes pas capables d'appliquer les textes que 
nous prenons dans des domaines aussi simples que les inscriptions, les contrôles des connaissances, l'octroi des bourses, comment pourrions-nous espérer gérer des réformes révolutionnaires?

Aujourd'hui, plus que jamais, dans le domaine de la formation comme dans d'autres, les Africains sont au pied du mur, face à leur destin.

\section{Arrêter des priorités}

La raréfaction des ressources financières consécutives à la crise économique qui dure depuis quinze ans, l'environnement international de moins en moins porté vers la pitié, la réduction progressive, et déjà perceptible, de l'aide publique au développement, nous contraignent à fixer rapidement des priorités.

En premier, il s'agit d'assurer l'éducation de base pour le plus grand nombre; une éducation de base qui soit le lieu d'apprentissages essentiels pour évoluer dans la vie moderne. Savoir lire, écrire, compter, représente de nos jours un minimum vital. Cette école fondamentale, comme nous l'avons appelée en Centrafrique en avril 1982, devrait également être le creuset de l'unité nationale qui fait si cruellement défaut à de nombreux pays africains.

Elle devrait être également le moule de la préparation de nouveaux Africains aptes à affronter les défis actuels du développement. C'est là que les jeunes Africains devraient apprendre à travailler dur, à valoriser l'effort et l'excellence, à respecter le bien public, à aimer leurs pays et à vouloir leur réussite, à pratiquer l'honnêteté et à acquérir le sens moral, en relation avec l'éducation familiale. C'est là qu'ils doivent assimiler les valeurs de la tolérance et de l'humanisme, et qu'ils peuvent s'ouvrir au monde contemporain en comprenant son rythme.

Par cette école de base, vitale pour le remodelage humain de l'Afrique, les soins les plus minutieux, l'attention et les sacrifices de tous sont requis. La qualité intellectuelle et morale des maîtres, la rigueur pédagogique et la discipline collective, l'exemple des adultes (parents, enseignants et administratifs), la bonne gestion des moyens sont la clé du succès.

51 On oublie trop souvent, par ailleurs, que la démocratisation tant souhaitée aujourd'hui, suppose des démocrates, c'est-à-dire, d'abord, des citoyens. Tout habitant d'une portion quelconque de la planète Terre n'est pas automatiquement un citoyen. Celui-ci, au contraire du simple habitant, s'identifie à une nation, à un pays dont il cultive l'amour. Le citoyen est celui qui accepte librement de contribuer à la préparation de l'avenir, et qui se sent de ce fait co-responsable de l'avenir commun.

Dans les années 1990-1992, quand s'enclencha le processus démocratique en Afrique, nous avons, on le sait, mimé la grande Révolution française, en organisant des « conférences nationales souveraines » qui se voulaient à l'image des États généraux de 1789. Nous aurions peut-être été mieux inspirés d'imiter aussi cette Révolution dans ce qu'elle a entraîné pour l'éducation, dans le lien que, très tôt, ses intellectuels, Condorcet en tête, ont perçu entre l'instruction et la formation du citoyen, entre l'instruction et l'exercice de la liberté. 


\section{Agir résolument}

Dans le contexte de la raréfaction des ressources financières, le débat sur le problème démographique en Afrique, en relation avec l'éducation, ne peut plus être escamoté comme nous l'avons fait jusqu'ici. Sous prétexte que le continent est sous-peuplé, la plupart des États africains considèrent le problème démographique comme une fausse querelle qui leur est faite. Mais pouvons-nous donc indéfiniment considérer comme un non-problème le fait que notre croissance économique n'arrive plus du tout à rattraper la croissance démographique, signifiant par-là que le progrès est définitivement renvoyé aux calendes grecques?

En d'autres termes, que faut-il choisir: une croissance démographique maîtrisée permettant d'améliorer la qualité de la vie pour le plus grand nombre, ou au contraire une population de plus en plus nombreuse, mais majoritairement analphabète, faute de moyens pour l'éducation, et de plus, misérable, composée à $75 \%$ de jeunes condamnés au chômage et à la révolte ? Faut-il accepter le développement tentaculaire de ces mégalopoles miséreuses, véritables poudrières sur lesquelles se construisent imprudemment les nouvelles institutions démocratiques africaines, avec des risques évidents d'explosions incontrôlables?

5 Le développement de l'enseignement de base, la garantie de sa qualité, ne sauraient évacuer ces questions.

Au-delà de ce cycle dont le développement se justifie, les États africains doivent se résoudre à accepter le fait qu'ils n'ont pas les moyens de généraliser l'enseignement secondaire, et encore moins le supérieur. Le mot "sélection » ne doit plus nous faire peur. La nécessité et le bon sens l'imposent désormais. Les efforts en faveur de l'enseignement de base doivent se faire en relation étroite avec le développement de filières de sortie, nécessairement courtes, profitables avant tout au monde rural, qui a tant besoin d'être revitalisé; profitables ensuite aux petits métiers urbains, sans lesquels il n'y a pas d'économie moderne.

57 Ne mentons pas à notre jeunesse en lui laissant croire que tout le monde est, dans les conditions actuelles, apte à poursuivre de longues études, surtout lorsqu'elles doivent déboucher sur le chômage massif.

Quant à nos universités, dont le discrédit est patent, elles doivent être débarrassées de tous ceux qui n'ont rien à y faire. Elles doivent devenir rapidement le lieu de la formation d'une élite responsable et exemplaire, et non la pépinière de délinquants en cols blancs.

Les solutions existent, et nous le savons tous, gouvernants, enseignants, parents. Il s'agit seulement d'agir résolument, sans attendre.

\section{NOTES}

1. «Braisage » : le fait de griller à la braise, au bûcher (argot estudiantin). 
2. Sango: langue vernaculaire, parlée et comprise dans tout le pays, et devenue la langue nationale de la République centrafricaine.

3. Si l'on avait été « repéré » en retard, en fin de sixième par exemple, des dispositions spéciales étaient prises pour un rattrapage en cinquième: cours particuliers, programmes intensifiés, enseignants spécialement désignés. Ce fut notre cas, en 1961-1962.

4. Axelle Kabou, Et si l'Afrique refusait le développement?, Éditions l'Harmattan, 1992, et notre opuscule Racines historiques et culturelles de la crise africaine, Abidjan, AD éditions et Cotonou, éditions du Pharaon, 1994.

5. PAS : programme d'ajustement structurel.

6. Les étudiants, rarement en manque de formules, ont baptisé le système des dérogations garanties du nom de " parapluie atomique », ce qui en dit long sur le sujet.

7. La crise de la dette, née de la déclaration du Mexique constatant, en 1981, son incapacité à rembourser les échéances de sa dette colossale, provoqua un véritable choc dans la communauté financière internationale. Elle ouvrit la porte aux négociations sur les rééchelonnements. Elle fut un élément décisif dans l'entrée en vigueur des PAS.

8. «On constate d'abord que ces programmes sont souvent élaborés selon un processus unidirectionnel. Des propositions sont formulées depuis Washington (Banque mondiale, Fonds monétaire), soumises à l'examen des gouvernements africains. Mais la conditionnalité qui accompagne ces propositions se traduit, de fait, par une situation de "à prendre ou à laisser ". Les États africains, pris financièrement à la gorge, n'ont donc pratiquement pas le choix. Le caractère technocratique de ces discussions, qui se mènent en vase clos entre experts, achève de convaincre les populations, sous informées, que tout cela est imposé par l'extérieur. » Jean-Paul Ngoupandé, Racines historiques et culturelles de la crise africaine, op.cit. p. 41. Et plus loin : «Mais l'interrogation qui à mes yeux est la plus importante, est la suivante : l'ajustement structurel n'est-il pas un mauvais rapiéçage consistant à poser un morceau de tissu neuf sur un ensemble obsolète? », op. cit. p. 42.

9. Crise africaine : l'électrochoc enfin ?, Conférence à Abidjan, 9 mars 1994, texte inédit.

10. «Cartouches » et "pétrole» désignent, dans l'argot estudiantin, des pratiques de fraudes auxquelles la majorité des élèves et étudiants recourent (notes cachées). Le «business » désigne l'entente occulte négociée avec des enseignants ou des responsables administratifs.

11. II est intéressant de noter que les sortants des lycées et collèges des pays africains francophones n'avaient, dans les années soixante et soixante-dix, aucun problème de niveau à leur arrivée dans les établissements d'enseignement supérieur français. Cette situation commença à évoluer négativement dans la seconde moitié des années soixante-dix. La dégradation du niveau est telle que des remises à niveau sont exigées depuis quelques années.

12. Dont près de $1 \%$ pour la seule Afrique du Sud

\section{RÉSUMÉS}

Pour l'auteur, l'incertitude et le désarroi moral qui pèsent sur les jeunes générations expriment un malaise "à multiples facettes" des sociétés africaines, confrontées à des difficultés économiques structurelles et, plus généralement, au choc culturel violent du passage à la modernité. Dans ce contexte, les enjeux d'une politique de formation exigeante sont vigoureusement soulignés. 
INDEX

Index géographique : Afrique subsaharienne

Mots-clés : comportement social, éthique, malaise de la jeunesse, système éducatif, valeur, éducation

\section{AUTEUR}

\section{JEAN-PAUL NGOUPANDÉ}

Ambassadeur de la Républicaine centrafricaine à Paris, ancien ministre cenrtrafricain de l'Éducation 